\title{
Bacillus Calmette-Guerin Antigen
}

National Cancer Institute

\section{Source}

National Cancer Institute. Bacillus Calmette-Guerin Antigen. NCI Thesaurus. Code

C77178.

An antigen preparation derived from Bacillus Calmette-Guerin, used as a component of BCG vaccine. 